\title{
Unlocking the Potential of Learning Analytics in Computing Education
}

\author{
SHUCHI GROVER, SRI International \\ ARI KORHONEN, Aalto University
}

\section{INTRODUCTION}

The following definition of learning analytics was used in the call for papers of the First International Conference on Learning Analytics and Knowledge (LAK 2011) and subsequently adopted by the Society for Learning Analytics (LA) Research: "the measurement, collection, analysis and reporting of data about learners and their contexts, for purposes of understanding and optimizing learning and the environments in which it occurs." However, the definition used by the late Erik Duval-"Learning Analytics is about collecting traces that learners leave behind and using those traces to improve learning"-is not only more simple and succinct but also makes clear the goal "to improve learning" while leaving open the kind of traces to be collected and how these traces are to be used to improve learning [1].

Big data and online learning have been among the main drivers pushing forward the field of LA. As with work being done in other disciplines, LA in computing education is closely intertwined with the sister field of educational data mining (EDM), with the latter focused more on the technical challenges of extracting meaning from data using data-driven techniques. Although EDM and LA have some foundational differences, the research in EDM and LA shares considerable overlap, and both communities are working toward the common goal of benefiting education and learners [2]. It appears that this is especially so in computing education, as evidenced in the collection of articles in this first volume of The Special issue on Learning Analytics in Computing Education.

The challenges and research questions are manifold: What background knowledge should students be assumed to have? How can we go about improving student retention? How do novices approach programming and debugging? How can we develop cognitive models of student programming knowledge? How can we evaluate novice programming environments? Do the attempts students make to solve an online programming exercise (or assignment) during the semester relate to their performance on final exam questions? Do assignments differentiate students who have mastered concepts from those who are still developing them? What factors get in the way of students' ability to demonstrate their knowledge?

\section{SERVING VARIED PURPOSES IN COMPUTING EDUCATION}

As the papers in this special issue suggest, learning analytics can be put to use to understand aspects of computing education that are important and yet too difficult, too time-consuming, too expensive, or simply not possible otherwise. These include understanding and measuring learning during the process of programming. In addition, some of the papers also emphasize the socio-cognitive aspects of learning computing, including understanding learners as part of a

Permission to make digital or hard copies of part or all of this work for personal or classroom use is granted without fee provided that copies are not made or distributed for profit or commercial advantage and that copies bear this notice and the full citation on the first page. Copyrights for third-party components of this work must be honored. For all other uses, contact the Owner/Author.

2017 Copyright is held by the owner/author(s).

ACM 1946-6226/2017/08-ART11e \$15.00

https://doi.org/10.1145/3122773 
participatory culture that includes social aspects such as how they interact with their peers during the programming process. Moreover, the future challenges appear to be ways of combining data from several sources and understanding better students' background knowledge, learning strategies, and motivations.

The first three articles contribute to learning analytics by focusing not only on reflections (what happened in the past) but also on predictions of students behavior in the future. Although the aim is to determine what kinds of interventions and feedback loops can be devised for the purpose of positively influencing their behavior, attitudes, and physiological state, the same learning data can also reveal weaknesses in learning environments and materials. Further, by continuously accumulating data generated as a by-product of students using various learning management systems, we can strategically intervene in the learning process. Indeed, this introduces an exciting direction for future computing education research.

The two last articles in this special issue are testament to the rapidly expanding role of CS education research into the K-12 space-a reality that was recently echoed in the editorial of the previous Transactions on Computing Education (TOCE) issue [4]. The articles herald the beginnings of learning analytics to understand various aspects of computing education that are unique to the K-12 arena-among others, the use of block-based environments for learning and the teaching of introductory programming as well as teen participation in computational activities in informal settings such as after-school programs and in online communities.

\section{OVERVIEW OF PAPERS}

The paper "IDE-Based Learning Analytics for Computing Education: A Process Model, Critical Review, and Research Agenda," by Christopher Hundhausen, Daniel Olivares and Adam Carter, is an excellent start to this special issue. Hundhausen et al. ground their study in four learning theories $[3,5,6,7]$ that they apply to the design of integrated development environments (IDEs) that are able to collect learning process data. They have augmented an IDE with a social networkingstyle activity stream, which automatically collects data on students' online conversations that take place in the same context as the programming activities. To facilitate the systematic exploration of this design space, the authors present an IDE-based data analytics process model with four primary activities: data collection, data analysis, intervention design, and intervention delivery. In addition, they conclude that the IDEs make it possible not only to collect learning process data but also to intervene in the learning process to create effective interventions grounded in learning theory. Finally, they present a call to action for future research into IDE-based learning analytics, including studies of privacy concerns, developing more unified research infrastructure and IDE facilities to collect and analyze a broader range of data, and designing and evaluating interventions based on theories of learning. Some of these aspects are already addressed in other papers in this special issue.

The next paper, "Blending Measures of Programming and Social Behavior into Predictive Models of Student Achievement in Early Computing Courses," by Adam Carter, Christopher Hundhausen, and Olusola Adesope, is a great follow-up to the previous one. Encouraged by social theories of learning, the authors incorporate a predictive measure derived from social behavior into three separate predictive measures derived from programming behaviors. These predictive models consisting of multiple factors associated with student success provide better predictive power than one single model alone. As the authors conclude, "This finding affirms the importance of social interaction in the learning process and provides evidence that predictive models derived from multiple sources of learning process data can provide significantly better predictive power by accounting for multiple factors responsible for student success." In this as well as in the next paper, the authors have utilized learning analytics to develop measures derived from learner data that 
significantly correlate with students' course grades. In addition, both papers acknowledge that measures derived from a single data source (e.g., social data or programming data) are likely to overlook explainable statistical variance. The authors therefore suggest that future development of predictive measures should consider the construction of more holistic models.

In the paper "A Contingency Table Derived Method for Analyzing Course Data" Alireza Ahadi, Arto Hellas, and Raymond Lister report on a study that explores the relationship between student performance on online exercises during a semester and subsequent performance on questions in a paper-based examination at the end of the semester. The authors conclude that variation of the students' performance in specific exam questions can be explained by the number of attempts a student makes on online exercises. The method devised for the measurement is based on constructing contingency tables. They identify exercises having high correlation with the final examination and, additionally, use the same method to identify students who will likely perform well or poorly. The method provides insight not only into which students would benefit from additional tutoring but also into problematic exercises that need attention. An important point of the article is to take into account many stakeholders who can benefit from looking at learner data (students, teachers, institutions, developers, researchers, etc.).

In "A Framework for Using Hypothesis-Driven Approaches to Support Data-Driven Learning Analytics in Measuring Computational Thinking in Block-Based Programming Environments," Shuchi Grover, Satabdi Basu, Marie Bienkowski, Michael Eagle, Nicholas Diana, and John Stamper draw attention to the need for understanding programming process instead of merely looking at the finished code or programs for evidence of computational thinking skills in the context of programming in block-based programming environments (BBPEs). They draw on exploratory analyses, including sequence mining on the Fairy Assessment dataset in the Alice BBPE [8] and a principled assessment design framework to present a blended "top-down"/"bottom-up" approach to better interpret patterns in log data that provide evidence of computational thinking (CT) during programming (including how programs are constructed through cycles of testing, construction, and debugging). They argue that in order to measure CT during programming, it is important to start with tasks that cover the spectrum of concepts and strategies needed for the performance being measured. They demonstrate the process through the construction of learner models for CT in the context of two programming tasks designed and piloted in high school Exploring Computer Science classrooms to help detect a priori patterns from log data. Such top-down approaches of pattern detection, when combined with data-driven ones, can be used in the future to provide appropriate and timely scaffolds to learner in "intelligent" BBPEs. Grover et al.'s research leverages the emerging realm of computational psychometrics, a technique that has shown promise in the context of game-based and simulation-based assessments in open-ended learning environments in science and math.

In the paper "Youth Computational Participation in the Wild: Understanding Experience and Equity in Participating and Programming in the Online Scratch Community," Deborah Fields, Yasmin Kafai, and Michael Giant systematically examine participation and computational creation in the context of informal learning by focusing on programmers in the popular Scratch community during a 3-month window of time. They characterize four levels of participation age and examine the quantity of programming concepts used in projects in relation to age and gender. In addition, they identify four classes or programming groups associated with increasing programming complexity-simple projects with mostly loops and no other constructs, more complex projects that used all concepts but no Booleans, projects that used all concepts including Booleans, and projects with greater use of all concepts and especially Booleans. Their findings reveal important lessons for computing education research-(1) the lowest programming complexity group was the largest and most stable (showed less fluidity in movement between groups over time window of the 
analysis), and (2) there were disproportionately high numbers of girls in the lowest programming complexity group and significantly more males than females in the group with highest programming complexity. This gender difference in the programming profile contrasts interestingly with their findings from an earlier analysis of the same dataset that revealed that there were no gender differences in indicators of participation as measured by the use of social features of the online Scratch community.

\section{REFERENCES}

[1] R. S. Baker, E. Duval, J. Stamper, D. Wiley, and S. B. Shum. 2012. Panel: Educational data mining meets learning analytics. In Learning Analytics and Knowledge. ACM.

[2] R. S. Baker and G. Siemens. 2014. Educational Data Mining and Learning Analytics. Cambridge Handbook of the Learning Sciences, K. Sawyer (Ed.).

[3] A. Bandura. 1986. Social Foundations of Thought and Action: A Social Cognitive Theory. Prentice Hall.

[4] C. Hundhausen. 2017. From the editor's desk: TOCE continues on a positive trajectory in 2016. ACM Trans. Comput. Educ. 17, 2, (2017), 5.

[5] J. Lave and E. Wenger. 1991. Situated Learning: Legitimate Peripheral Participation. Cambridge University Press.

[6] J. B. Rotter. 1966. Generalized expectancies for internal versus external control of reinforcement. Psychol. Monogr. Gen. Appl. 80, 1, (1966), 1-28.

[7] J. Sweller, J. J. Van Merrienboer, and F. G. W. Paas. 1998. Cognitive architecture and instructional design. Educ. Psychol. Rev. 10, 3, (1998), 251-296.

[8] L. Werner, J. Denner, S. Campe, and D. C. Kawamoto. 2012. The Fairy Performance Assessment: Measuring Computational Thinking in Middle School. In Proceedings of the 43rd ACM Technical Symposium on Computer Science Education. 215-220. New York, NY: ACM (2012). 